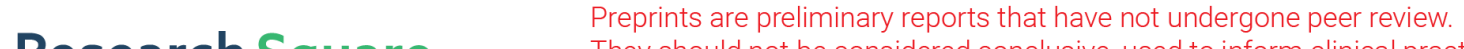 They should not be considered conclusive, used to inform clinical practice, or referenced by the media as validated information. \\ Clinical implication and immunological landscape analyses of ANLN in pan-cancer, a new target for cancer research
}

\section{Lan Zhang}

Shanghai Tenth People's Hospital

\section{Yong Wei}

Second Affiliated Hospital of Nanjing Medical University

\section{Yan $\mathrm{He}$}

The Affiliated Suzhou Hospital of Nanjing Medical University

\section{Xiaping Wang}

Second Affiliated Hospital of Nanjing Medical University

\section{Zebo Huang}

Wuxi Fourth People's Hospital

\section{Libing Sun}

The Affiliated Suzhou Hospital of Nanjing Medical University

Jie Chen

Dushu Lake Hospital Affiliated to Soochow University (Medical Center of Soochow University)

\section{Qingyi Zhu}

Second Affiliated Hospital of Nanjing Medical University

\section{Xin Zhou ( $\nabla$ zhouxin5523@jsph.org.cn )}

First Affiliated Hospital of Nanjing Medical University

\section{Research Article}

Keywords: ANLN, pan-cancer, prognosis, immune infiltration, TMB

Posted Date: February 16th, 2022

DOI: https://doi.org/10.21203/rs.3.rs-1273014/v1

License: (c) (i) This work is licensed under a Creative Commons Attribution 4.0 International License. Read Full License 


\section{Abstract \\ Background}

Actin-binding protein anillin (ANLN) is mainly involved in the process of cytokinesis and known to be dysregulated in diverse cancers. However, the role of ANLN in pan-cancer prognosis and tumor immunity remains unclear.

\section{Methods}

Gene expression profiles of 31 solid tumors were fetched from The Cancer Genome Atlas (TCGA) database. ANLN mRNA and protein expression were quantified via quantitative real-time PCR (qRT-PCR) and immunohistochemistry (IHC). Protein expression of ANLN was further confirmed in Human Protein Atlas (HPA) database. Cox regression and Kaplan-Meier analysis were utilized to assess the prognostic value of ANLN in different tumors. The association between ANLN and different immune gene markers and infiltration cells were analyzed via ESTIMATE and CIBERSORT. A BLCA immunotherapy cohort: IMvigor (210) was utilized to confirm the role of ANLN in immune response.

\section{Results}

ANLN upregulation was detected in 21 types of cancers and was correlated with poor overall survival (OS), disease-free interval (DFI) and progression-free interval (PFI) in most cancers except in THYM. Additionally, correlation analysis revealed a significant association between ANLN expression and tumor mutation burden (TMB), microsatellite instability (MSI), immune cells infiltration and immune checkpoint genes in most cancer types. A BLCA immunotherapy cohort confirmed that patients with higher ANLN level had better immune responses and longer OS.

\section{Conclusions}

ANLN may serve as a prognostic biomarker for pan-cancer. ANLN upregulation is associated with higher TMB, MSI and immune cell infiltration in multiple types of tumors, shedding new light for cancer treatment.

\section{Background}

Anillin (ANLN) is a F-actin binding protein originally isolated from Drosophila embryo [1]. It is highly conserved and mainly involved in the cortical cytoskeletal dynamics during cytokinesis and cellularization [2]. Dysregulations of ANLN is observed in a wide range of malignant tumors and its overexpression is closely linked to unfavorable outcomes in colorectal cancer [3], breast cancer [4] and liver cancer [5]. However, the role of ANLN in pan-cancer remains still unclear. 
The tumor microenvironment (TME) consists of not only cancer cells, but also tumor-associated normal cells, including infiltrating stromal and immune cells [6]. Increasing evidence suggests that TME has multiple effects on tumorigenesis, development and prognosis [7-10]. However, the possible mechanisms of stromal and immune components remain elusive. Immunotherapy has proved to be a powerful clinical strategy for cancer treatment [11]. Monoclonal antibodies targeting cytotoxic $T$ lymphocytes associated antigen 4 (CTLA4), programmed death-1 (PD-1) receptor and its ligand (PD-L1) have achieved clinical success in some cancers [12-14]. However, only a subset of patients could benefit from current immunotherapies [15]. Thus, the discovery of effective immunotherapy targets is of great clinical significance.

Hence, our study comprehensively explored the prognostic significance of ANLN in 31 tumors based on TCGA data. Subsequently, we explored the potential correlations between ANLN and tumor immune characteristics.

\section{Methods}

\section{Data Collection and Preprocessing}

Gene expression profiles of 31 types of solid tumors including raw counts and Fragments Per Kilobase of transcript per Million (FPKM)-normalized RNA-seq data were downloaded from The Cancer Genome Atlas (TCGA) database (https://portal.gdc.cancer.gov/) on April 20 2021. Acute myeloid leukemia (LAML) and large B-cell lymphoma (DLBC) were excluded because they were hematological tumors. The primary data is shown in table 1. Protein-encoding genes were aligned to the human reference genome assembly GRCh38. Transcriptome files of 18,321 related genes were normalized with the assistance from R packages 'limma' on R platform v3.6.3. and converged into a matrix for further analysis. The difference in ANLN expression was compared between normal tissues and tumor tissues using the Wilcoxon test with a threshold of $P<0.05$. ANLN protein expression data in 16 types of cancers were retrieved via the Human Protein Atlas (HPA) database (https://www.proteinatlas.org/).

\section{Prognosis Analysis}

The prognostic values of ANLN, including Overall Survival (OS) and Disease-Free Interval (DFI) and Progression-Free Interval (PFI) was assessed via univariate Cox regression model. Samples were clustered into high or low expression cohorts based on the median value of ANLN. Kaplan-Meier (K-M) curves and the forest plots were utilized to compare the survival time differences. All analyses were carried out using the 'survival 'and 'survminer' package. After that, the associations between ANLN and clinical parameters, including age, sex and the TNM stage were assessed by the chi-square test or Fisher's exact test. All tests were two-tailed and a p-value of 0.05 or less was considered statistically significant.

\section{Correlation between ANLN expression and TMB and MSI}


Tumor mutational burden (TMB) is a measure of the amount of somatic coding mutations per DNA megabase [16]. Microsatellite instability (MSI) is a molecular tumor phenotype of a deficient mismatch repair system [17]. Analysis regarding the correlation between ANLN expression and TMB/MSI was conducted by Pearson correlation analysis and shown in the radar chart using the 'fmsb' package.

\section{Correlation between ANLN expression and tumor immunity}

ESTIMATE (Estimation of Stromal and Immune cells in Malignant Tumor tissues using Expression data) is a method for estimating the presence of stromal/immune cells in tumor tissues and tumor purity with high accuracy [18]. This method was applied to explore the association between the immune and stromal cell ratio with ANLN expression [18].

We further explored the correlation between ANLN expression and 22 types of infiltrating immune cells using the 'CIBERSORT' algorithm. The assiciation between ANLN expression and common immune checkpoint genes was investigated using the 'Reshape2' package and shown in heatmaps by the $\mathrm{R}$ 'RColorBrewer' package. The Spearman method was utlized to assess the correlation coefficients.

\section{Collection of an immunotherapy-based cohort}

After systematically searching for immunotherapy gene expression profiles, an immunotherapeutic cohort: advanced urothelial cancer with intervention of atezolizumab, an anti-PD-L1 antibody (IMvigor210 cohort) with complete clinical information was recruited in our study [19]. The complete information of the cohort is available on http://research-pub.Gene.com/imvigor210corebiologies. The raw count data were normalized by the DEseq2 R package and transformed into the TPM value for further study.

\section{Gene Set Enrichment Analysis}

Next, our study explored the potential molecular functions and biological pathways regulated by ANLN. The Gene Set Enrichment Analysis (GSEA) software (http://www.broadinstitute. org/gsea) was used to investigate the enrichment of GO and KEGG pathways between high ANLN expression and low expression groups. All analyses were conducted with R package 'org.Hs.eg.db', 'clusterProfiler', 'enrichplot', 'DOSE /limma'. Gene sets with $|\mathrm{NES}|>1$, NOM $p<0.05$, and FDR $q<0.25$ were considered as enrichment significant.

\section{Experimental Validation}

Formalin-fixed paraffin-embedded (FFPE) tumor and para-tumor tissue samples of 7 LUAD patients were collected from The Affiliated Suzhou Hospital of Nanjing Medical University. The mRNA and protein expression of ANLN were quantified using quantitative real-time PCR (qRT-PCR) and immunohistochemistry (IHC) as previously described[10]. GAPDH was utilized as an internal control in qRT-PCR. The sequences of the primers used were as follows: forward: 5'-

CAGACAGTTCCATCCAAGGGAG-3' and reverse: 5'-CTTGACAACGCTCTCCAAAGCG-3'. This study was 
approved by the Ethics Committee of the hospital. Each participant had signed informed consent in accordance with the Declaration of Helsinki. (Ethics Approval No. KL901198).

\section{Results}

\section{Pan-cancer ANLN expression}

Our study firstly analyzed the expression pattern of ANLN in tumor and normal samples from the TCGA pan-cancer dataset. As shown in Figure 1A, ANLN expression was significantly increased in BLCA, BRCA, CESC, CHOL, COAD, ESCA, HNSC, KICH, KIRC, KIRP, LIHC, LUAD, LUSC, PAAD, PCPG, PRAD, READ, SARC, STAD, THCA and UCEC. Next, we investigated ANLN protein expression based on the HPA database. ANLN expression was significantly higher in tumor samples than normal tissues in BLCA, CRC (including COAD and READ), LUAD, OV and TGCT (Figure 1B). We further validated ANLN expression in LUAD patients. The immunohistochemical and mRNA results demonstrated that ANLN expression was significantly higher in tumor tissues than paracancerous samples (Figure 1C, 1D).

\section{Survival analysis of ANLN}

Our study investigated the correlation between ANLN expression and patients' OS, DFI, PFI using the TCGA cohort. Kaplan-Meier curves showed that high expression of ANLN was significantly associated with poor OS in ACC, BLCA, KICH, KIRC, KIRP, LIHC, LUAD, MESO, PAAD, PCPG and PRAD. Particularly, low expression of ANLN was linked to poor OS in THYM. In addition, high ANLN expression was correlated with poor DFI in BRCA, KIRP, LIHC, LUAD, PAAD and THCA. Results of the Kaplan-Meier plot also suggested that increased level of ANLN was distinctly connected with worse PFI in patients with ACC, BLCA, KIRC, KIRP, LIHC, LUAD, MESO, PAAD, PCPG, PRAD, UCEC and UVM (Figure 2).

In Cox regression analysis, high ANLN expression was an unfavorable factor for patients' OS in ACC, BLCA, CESC, CHOL, KICH, KIRC, KIRP, LIHC, LUAD, MESO, PAAD, PCPG, UCEC and UVM. While patients with low ANLN expression showed shortened OS in THYM. For DFI, elevated level of ANLN was significantly correlated with shorter DFI in BRCA, KIRP, LIHC, LUAD, PAAD, PRAD and THCA. Additionally, increased level of ANLN was strongly correlated with worse PFI in ACC, BLCA, HNSC, KICH, KIRC, KIRP, LIHC, LUAD, MESO, PAAD, PCPG, PRAD, THCA, UCEC and UVM (Figure 3).

Furthermore, we explored the correlation between ANLN Expression and different clinical features across pan-cancer. Results revealed that higher expression of ANLN was significantly associated with advanced TNM stage in ACC, BLCA, BRCA, ESCA, KICH, KIRC, KIRP, LUAD and reversely in LIHC. Interestingly, ANLN expression was higher in stage I compared to stage II $(P=0.008)$, but lower in stage Il compared to stage IV $(P=0.0018)$ in THCA (Figure 4). ANLN expression also differs greatly in age and gender across different tumors (Figure S1).

\section{Tumor mutational burden and microsatellite instability analysis data}


Tumor mutational burden (TMB) and microsatellite instability (MSI) are essential in the process of tumor formation and development. We assessed the association between ANLN expression and TMB/MSI across 31 cancers. Results suggested that elevated ANLN expression was significantly associated with higher TMB in ACC, BLCA, BRCA, COAD, KICH, KIRC, LUAD, LUSC, MESO, PAAD, PRAD, READ, SARC, SKCM, STAD, TGCT, THCA and UCEC, while increased ANLN level was inversely related to TMB in LGG and THYM (Figure 5). In addition, ANLN expression was positively associated with MSI in ACC, COAD, LIHC, LUSC, MESO, READ, SARC, STAD, UCEC and negatively linked to SKCM (Figure 5).

\section{Correlation between ANLN expression and immune infiltration}

Tumor-infiltrating immune cells are important components for the tumor microenvironment, and are closely linked to tumorigenesis and progression [20]. Infiltrating stromal and immune cells in tumor microenvironment play essential roles in disturbing tumor signaling and cancer biology [6]. Hence, we firstly explored the correlation between ANLN expression and tumor-infiltrating immune cells. Our results demonstrated that ANLN expression was significantly associated with the infiltration levels

of macrophages M1 in 18 cancer types (Figure 6, those with $P<0.00001$ were shown), CD4+T cells in 12 cancer types, CD8+ T cells in 9 cancer types, Tregs in 13 cancer types, B cells in 8 cancer types, M2 in 7 cancer types, NK cells in 9 cancer types and neutrophils in 7 cancer types (those with $P<0.00001$ were shown in Figure S2). For instance, in BRCA, ANLN expression was negatively correlated with macrophages M2, monocytes, but positively correlated with macrophages M1, T cells CD4 memory activated, T cells follicular helper. In HNSC, ANLN expression was negatively correlated with CD8+ cells, Tregs, but positively correlated with $\mathrm{CD} 4+\mathrm{T}$ cells memory resting.

Secondly, our study investigated the association between ANLN expression and the immune and stromal cell ratio. Results showed that ANLN expression is positively correlated with the stromal score and immune score in KIRC, THCA, while negatively correlated with in LUSC, SARC, STAD and UCEC (Figure 7). In addition, our study observed a statistical positive connection between ANLN expression and stromal score in PRAD but noted a negative connection in BRCA, LIHC, TGCT. Moreover, ANLN expression was negatively linked to immune score in PAAD and SKCM (Figure 7).

Immune checkpoint genes (ICGs) represent a novel target in cancer treatment. Therefore, we further examined the association between ANLN expression and the common immune checkpoint genes. As shown in Figure 8, cytotoxic T lymphocyte-associated antigen 4 (CTLA-4), programmed death 1 (PD-1) and PD-L1 was positively associated with ANLN expression in multiple cancers, including BRCA, KICH, KIRC, LIHC, PRAD and THCA. On the contrary, there was a negative connection between ANLN expression and CTLA-4, PD-1 in CESC, HNSC and LUSC. Moreover, ANLN expression was positively linked to the majority of immune checkpoint genes in THCA. However, ANLN expression demonstrated a negative correlation with most of the immune checkpoint genes in GBM, UCS and UVM.

\section{Immunotherapeutic Response Prediction}


Considering the strong correlation between ANLN expression and tumor immune landscape, our study next explored the immunotherapeutic predictive ability of ANLN in an anti-PD-L1 cohort (IMvigor210). The Kaplan-Meier curves suggested that increased level of ANLN was significantly connected with prolonged OS (Figure 9A). In addition, patients with higher ANLN expression level exhibited better immune response (Figure 9B, C).

\section{GO and KEGG based Gene Set Enrichment Analysis}

Next, GSEA was applied to explore the functional enrichment of ANLN expression between high expression and low expression cohorts. GO enrichment term demonstrated that high expression of ANLN was mainly associated with cell immunity and cell proliferation Figure S3). KEGG enrichment term exhibited that high expression of ANLN was positively involved 'cell cycle' pathways in ACC, BRCA, KICH, HHSC, KICH, LUAD. MESO, OV, PAAD, PCPG, SARC and UVM (Figure S4).

\section{Discussion}

The pan-cancer analysis project launched by The Cancer Genome Atlas (TCGA) was aimed to illustrate an integrated picture of commonalities and heterogeneities across different tumors [21]. The project interpreted molecular aberrations from genomic, transcriptional, proteomic and epigenetic levels in large patient cohorts, providing comprehensive information for early diagnosis and treatment strategy [22]. Our study is the first to comprehensively analyze the expression pattern of ANLN and its correlation with patients' prognosis and immune features in 31 different types of cancers.

Our study found that ANLN was upregulated in BLCA, BRCA, CESC, CHOL, COAD, ESCA, HNSC, KICH, KIRC, KIRP, LIHC, LUAD, LUSC, PAAD, PCPG, PRAD, READ, SARC, STAD, THCA and UCEC. Cox regression and Kaplan-Meier analysis demonstrated that high ANLN expression was associated with shorter OS, DFI and PFS in KIRP, LIHC, LUAD and PAAD. This is in line with a recent study that ANLN is a prognostic biomarker for LUAD patients [23]. ANLN is primarily involved in the process of cytokinesis and plays vital roles in tumorigenesis and progression[2]. ANLN could significantly promote cancer cell growth and migration via targeting miR-217 [24] and HMGA2 [25]. Up-regulation of ANLN could activate RHOA, leading to the activation of phosphatidylinositol 3-kinase-AKT (PI3K/AKT), which promotes cell proliferation and progression [26]. Another study revealed that ANLN might be involved in the metastasis of lung adenocarcinoma by promoting epithelial mesenchymal transformation of tumor cells [27]. In addition, ANLN expression is also positively related to $\mathrm{Wnt} / \beta$-catenin signaling in gastric cancer [28]. All these biological functions of ANLN may explain its oncogenic role in the above-mentioned cancers. Notably, over-expression of ANLN was correlated with better prognosis in THYM. Future studies are needed to explore its controversial role in human cancer.

TMB is emerging as a promising biomarker for immunotherapy. It refers to the total number of somatic coding mutations, gene insertion, base substitutions and deletion errors detected per million bases [29]. TMB presents as a useful estimation of tumor neoantigens, which could activate tumor-specific $T$ cells to recognize and kill tumor cells [29]. High TMB are associated with prolonged survival in melanoma [30], 
NSCLC [31] and breast cancer [32]. MSI is a molecular tumor phenotype caused by genomic hypermutability [33]. Recent studies suggested that MSI could serve as an efficient biomarker for immunotherapy [34]. Clinical trials showed that MSI-H patients have better outcomes than microsatellite stable patients, owning to the activation of T lymphocyte recognition of neoantigens [34,35]. Additionally, nearly all patients with MSI-H have high TMB level and could respond more strongly to immunotherapy [16]. Our study found that ANLN expression was positively associated with TMB or MSI in multiple cancers. This suggested that ANLN expression in these tumors may help identify patients who are more likely to benefit from immunotherapy.

TME is a complex ecology consisting of tumor cells, tumor vasculature, surrounding immune and inflammatory cells, as well as adipocytes and fibroblasts [6]. Tumor immune microenvironment (iTME), as a core part of the TME, consists of tumor-infiltrating lymphocytes (TILs) and other immune cells, such as macrophages, neutrophils and dendritic cells [36]. TILs, including B cells and T cells, are widely accepted as the most crucial effectors during the host anti-tumor immune response and prognosis [20]. We found that ANLN expression was negatively connected to CD8+ T cells in some cancers. Cytotoxic CD8+ memory T cells in the TME is positively linked to cancer prognosis in diverse cancers [37-39]. It could contribute to cancer prognoses by killing tumor cells [40]. We speculated that ANLN might help immune escape by reducing CD8+ T cell infiltration. Further studies are needed to validate the association between ANLN expression and CD8+ T cells. Macrophages is a group of heterogeneous cells in the iTME and is strongly correlated with poor survival in diverse cancers [41]. Tumor-associated macrophages (TAMs) play essential roles in carcinogenesis and progression by providing cytokines and inducing tumor angiogenesis [42]. TAMs could be polarized into two distinct phenotypes: 1) M1 macrophages, which act as an anti-tumor defender; 2) M2 macrophages, which could promote tumor invasion and metastasis [42]. Our study found that ANLN expression was positively associated with M1 macrophage infiltration, but negatively linked to M2 macrophage infiltration. This could be explained by the existence of other important regulators in the interaction between ANLN and TAMs. Further studies are needed to investigate the exact mechanism during this anti-tumor progress. Single-cell RNA sequencing (scRNA-seq), which allows for transcriptome-wide analyses of individual cells may help offer new insights in the future. We also found there was a positive connection between ANLN expression and neutrophils in some cancers. Tumor-associated neutrophils are commonly recognized as tumorpromoting agents in malignant tumors by initiating and promoting inflammatory response [43]. Thus, we assumed that ANLN might participate in tumor progression by inducing neutrophil infiltration. A previous study showed that ANLN along with other regulators are significantly associated with the expression of naïve B cells, regulatory T cells and neutrophils in head and neck squamous cell carcinoma (HNSCC) [44]. However, studies focusing on the functional mechanism of ANLN in immune cell infiltration is rare. Our GO and KEGG pathway analyses revealed that ANLN was mainly enriched in cell cycle, immunity and metabolic-related pathway, which may explain its oncogenic role in different tumors. Further studies are needed to find the exact functional mechanism between ANLN expression and different immune cells. Moreover, our analyses demonstrated that ANLN expression had a significant correlation with most of the common immune checkpoint markers. We further validated the immunotherapeutic predictive ability of 
ANLN in an anti-PD-L1 cohort (IMvigor210). Results showed that high ANLN expression was associated with prolonged $\mathrm{OS}$ and better immune response, which was consistent with the above definitions. All these above findings confirmed our hypothesis that ANLN could be a robust biomarker for prognosis and immune response prediction.

Several limitations should be considered in our study. First, because of the use of microarray and sequencing data from different databases, systematic bias may occur. Hence, as mentioned above, scRNA-seq or other higher resolution methods could be performed. Second, our study was a retrospective study, so future prospective researches are needed to verify the correlation between ANLN expression and patients' prognosis and tumor immunity. Finally, the precise mechanisms by which ANLN facilitates tumor progression and tumor immunity remain largely obscure. Further mechanistic studies on ANLN at both cellular and molecular levels are needed to fully elucidate its functions.

\section{Conclusions}

Our study found that ANLN was upregulated in diverse cancers and its aberrant expression was associated with pan-cancer prognosis, MSI, TMB, the tumor immune microenvironment and immune checkpoint genes. Therefore, ANLN may prove to be a promising biomarker for prognostic judgment and immunotherapy.

\section{Abbreviations}

ANLN: anillin;

TME: tumor microenvironment;

CTLA4: cytotoxic T lymphocytes associated antigen 4;

PD-1: programmed death-1;

PD-L1: receptor and its ligand;

TMB: tumor mutation burden;

MSI: microsatellite instability;

FPKM: Fragments Per Kilobase of transcript;

TCGA: The Cancer Genome Atlas;

ACC: adrenocortical carcinoma;

BLCA: bladder urothelial carcinoma; 
BRCA: breast invasive carcinoma;

CESC: cervical squamous cell carcinoma;

CHOL: cholangiocarcinoma;

COAD: colon adenocarcinoma;

DLBC: lymphoid neoplasm diffuse large B-cell lymphoma;

ESCA: esophageal carcinoma;

GBM: glioblastoma multiforme;

LGG: brain lower grade glioma;

HNSCC: head and neck squamous cell carcinoma;

$\mathrm{KICH}$ : kidney chromophobe;

KIRC: kidney renal clear cell carcinoma;

KIRP: kidney renal papillary cell carcinoma;

LIHC: liver hepatocellular carcinoma;

LUAD: lung adenocarcinoma;

LUSC: lung squamous cell carcinoma;

MESO: mesothelioma;

OV: ovarian serous cystadenocarcinoma;

PAAD: pancreatic adenocarcinoma;

PCPG: pheochromocytoma and paraganglioma;

PRAD: prostate adenocarcinoma;

READ: rectum adenocarcinoma;

SARC: sarcoma;

SKCM: skin cutaneous melanoma;

STAD: stomach adenocarcinoma; 
TGCT: testicular germ cell tumors;

THCA: thyroid carcinoma;

THYM: thymoma;

UCEC: uterine corpus endometrial carcinoma;

UCS: uterine carcinosarcoma;

UVM: uveal melanoma;

LAML: acute myeloid leukemia;

DLBC: large B-cell lymphoma;

HPA: Human Protein Atlas;

OS: overall survival;

DFI: disease-free interval;

PFI: progression-free interval;

K-M curves: Kaplan-Meier curves;

ESTIMATE: estimation of Stromal and Immune cells in Malignant Tumor tissues using Expression data;

TFH cells: T follicular helper cells;

Tregs: regulatory T cells;

GSEA: The Gene Set Enrichment Analysis;

FFPE: Formalin-fixed paraffin-embedded;

qRT-PCR: quantitative real-time PCR;

IHC: immunohistochemistry;

ITME: tumor immune microenvironment;

TILs: tumor-infiltrating lymphocytes;

TAMs: tumor-associated macrophages;

scRNA-seq: single-cell RNA sequencing; 


\section{Declarations}

\section{Acknowledgements}

Not applicable.

\section{Funding}

This work was supported by the National Natural Science Foundation of China [Grant number: 81772732; 81702364] and the Natural Science Foundation of Jiangsu Province [Grant number: BK20171085].

\section{Availability of data and materials}

The data that support the findings of this study are available from the corresponding author upon reasonable request.

\section{Ethics approval and consents to participate}

This study was approved by the Ethics Committee of The Affiliated Suzhou Hospital of Nanjing Medical University and each participant had signed informed consent (Ethics Approval No. KL901198).

\section{Competing interests}

The authors declare that they have no competing interests.

\section{Consent for publication}

Not applicable.

\section{Author Contributions}

JC, QYZ and XZ conceived and designed this study. LZ, YW and YH obtained the data. XZ, LZ, YW and YH analyzed the data. XPW and ZBH helped discuss the results. LZ drafted the manuscript. All authors contributed to the article and approved the submitted version.

\section{References}

1. Tuan NM, Lee CH: Role of Anillin in Tumour: From a Prognostic Biomarker to a Novel Target. Cancers (Basel) 2020, 12(6).

2. Chen A, Akhshi TK, Lavoie BD, Wilde A: Importin beta2 Mediates the Spatio-temporal Regulation of Anillin through a Noncanonical Nuclear Localization Signal. J Biol Chem 2015, 290(21):1350013509. 
3. Wang G, Shen W, Cui L, Chen W, Hu X, Fu J: Overexpression of Anillin (ANLN) is correlated with colorectal cancer progression and poor prognosis. Cancer Biomark 2016, 16(3):459-465.

4. Dai X, Mei Y, Chen X, Cai D: ANLN and KDR Are Jointly Prognostic of Breast Cancer Survival and Can Be Modulated for Triple Negative Breast Cancer Control. Front Genet 2019, 10:790.

5. Zhou Z, Li Y, Hao H, Wang Y, Zhou Z, Wang Z, Chu X: Screening Hub Genes as Prognostic Biomarkers of Hepatocellular Carcinoma by Bioinformatics Analysis. Cell Transplant 2019, 28(1_suppl):76S-86S.

6. Fridman WH, Pages F, Sautes-Fridman C, Galon J: The immune contexture in human tumours: impact on clinical outcome. Nat Rev Cancer 2012, 12(4):298-306.

7. Ge Q, Li G, Chen J, Song J, Cai G, He Y, Zhang X, Liang H, Ding Z, Zhang B: Immunological Role and Prognostic Value of APBB1 IP in Pan-Cancer Analysis. J Cancer 2021, 12(2):595-610.

8. Liu J, Zhang S, Dai W, Xie C, Li JC: A Comprehensive Prognostic and Immune Analysis of SLC41A3 in Pan-Cancer. Front Oncol 2020, 10:586414.

9. Xu WX, Zhang J, Hua YT, Yang SJ, Wang DD, Tang JH: An Integrative Pan-Cancer Analysis Revealing LCN2 as an Oncogenic Immune Protein in Tumor Microenvironment. Front Oncol 2020, 10:605097.

10. Yang C, Wu T, Zhang J, Liu J, Zhao K, Sun W, Zhou X, Kong X, Shi J: Prognostic and Immunological Role of mRNA ac4C Regulator NAT10 in Pan-Cancer: New Territory for Cancer Research? Front Oncol 2021, 11:630417.

11. Conway JR, Kofman E, Mo SS, Elmarakeby H, Van Allen E: Genomics of response to immune checkpoint therapies for cancer: implications for precision medicine. Genome Med 2018, 10(1):93.

12. Gadgeel S, Rodriguez-Abreu D, Speranza G, Esteban E, Felip E, Domine M, Hui R, Hochmair MJ, Clingan P, Powell SF et al: Updated Analysis From KEYNOTE-189: Pembrolizumab or Placebo Plus Pemetrexed and Platinum for Previously Untreated Metastatic Nonsquamous Non-Small-Cell Lung Cancer. J Clin Oncol 2020, 38(14):1505-1517.

13. Kato K, Shah MA, Enzinger P, Bennouna J, Shen L, Adenis A, Sun JM, Cho BC, Ozguroglu M, Kojima T et al: KEYNOTE-590: Phase III study of first-line chemotherapy with or without pembrolizumab for advanced esophageal cancer. Future Oncol 2019, 15(10):1057-1066.

14. Andre T, Shiu KK, Kim TW, Jensen BV, Jensen LH, Punt C, Smith D, Garcia-Carbonero R, Benavides M, Gibbs P et al: Pembrolizumab in Microsatellite-Instability-High Advanced Colorectal Cancer. N Engl J Med 2020, 383(23):2207-2218.

15. Topalian SL, Drake CG, Pardoll DM: Immune checkpoint blockade: a common denominator approach to cancer therapy. Cancer Cell 2015, 27(4):450-461.

16. Chalmers ZR, Connelly CF, Fabrizio D, Gay L, Ali SM, Ennis R, Schrock A, Campbell B, Shlien A, Chmielecki $\mathrm{J}$ et al: Analysis of 100,000 human cancer genomes reveals the landscape of tumor mutational burden. Genome Med 2017, 9(1):34.

17. Thibodeau SN, Bren G, Schaid D: Microsatellite instability in cancer of the proximal colon. Science 1993, 260(5109):816-819. 
18. Yoshihara K, Shahmoradgoli M, Martinez E, Vegesna R, Kim H, Torres-Garcia W, Trevino V, Shen H, Laird PW, Levine DA et al: Inferring tumour purity and stromal and immune cell admixture from expression data. Nat Commun 2013, 4:2612.

19. Mariathasan S, Turley SJ, Nickles D, Castiglioni A, Yuen K, Wang Y, Kadel EE, III, Koeppen H, Astarita $\mathrm{JL}$, Cubas $\mathrm{R}$ et al: TGFbeta attenuates tumour response to PD-L1 blockade by contributing to exclusion of T cells. Nature 2018, 554(7693):544-548.

20. Savas P, Salgado R, Denkert C, Sotiriou C, Darcy PK, Smyth MJ, Loi S: Clinical relevance of host immunity in breast cancer: from TILs to the clinic. Nat Rev Clin Oncol 2016, 13(4):228-241.

21. Cancer Genome Atlas Research N, Weinstein JN, Collisson EA, Mills GB, Shaw KR, Ozenberger BA, Ellrott K, Shmulevich I, Sander C, Stuart JM: The Cancer Genome Atlas Pan-Cancer analysis project. Nat Genet 2013, 45(10):1113-1120.

22. Consortium ITP-CAoWG: Pan-cancer analysis of whole genomes. Nature 2020, 578(7793):82-93.

23. Song C, Wu Z, Wang Q, Wang Y, Guo Z, Li S, Hu W: A Combined Two-mRNA Signature Associated With PD-L1 and Tumor Mutational Burden for Prognosis of Lung Adenocarcinoma. Front Cell Dev Biol 2021, 9:634697.

24. Idichi T, Seki N, Kurahara H, Yonemori K, Osako Y, Arai T, Okato A, Kita Y, Arigami T, Mataki Y et al: Regulation of actin-binding protein ANLN by antitumor miR-217 inhibits cancer cell aggressiveness in pancreatic ductal adenocarcinoma. Oncotarget 2017, 8(32):53180-53193.

25. Guo HH, Wang YZ, Zhang ZK, Li MZ, Tian XD, Yang YM: High mobility group AT-hook 2 promotes tumorigenicity of pancreatic cancer cells via upregulating ANLN. Exp Cell Res 2020, 393(1):112088.

26. Suzuki C, Daigo Y, Ishikawa N, Kato T, Hayama S, Ito T, Tsuchiya E, Nakamura Y: ANLN plays a critical role in human lung carcinogenesis through the activation of RHOA and by involvement in the phosphoinositide 3-kinase/AKT pathway. Cancer Res 2005, 65(24):11314-11325.

27. Xu J, Zheng H, Yuan S, Zhou B, Zhao W, Pan Y, Qi D: Overexpression of ANLN in lung adenocarcinoma is associated with metastasis. Thorac Cancer 2019, 10(8):1702-1709.

28. Pandi NS, Manimuthu M, Harunipriya P, Murugesan M, Asha GV, Rajendran S: In silico analysis of expression pattern of a Wnt/beta-catenin responsive gene ANLN in gastric cancer. Gene 2014, 545(1):23-29.

29. Samstein RM, Lee CH, Shoushtari AN, Hellmann MD, Shen R, Janjigian YY, Barron DA, Zehir A, Jordan EJ, Omuro A et al: Tumor mutational load predicts survival after immunotherapy across multiple cancer types. Nat Genet 2019, 51(2):202-206.

30. Kang K, Xie F, Mao J, Bai Y, Wang X: Significance of Tumor Mutation Burden in Immune Infiltration and Prognosis in Cutaneous Melanoma. Front Oncol 2020, 10:573141.

31. Gandara DR, Paul SM, Kowanetz M, Schleifman E, Zou W, Li Y, Rittmeyer A, Fehrenbacher L, Otto G, Malboeuf $C$ et al: Blood-based tumor mutational burden as a predictor of clinical benefit in nonsmall-cell lung cancer patients treated with atezolizumab. Nat Med 2018, 24(9):1441-1448.

32. Thomas A, Routh ED, Pullikuth A, Jin G, Su J, Chou JW, Hoadley KA, Print C, Knowlton N, Black MA et al: Tumor mutational burden is a determinant of immune-mediated survival in breast cancer. 
Oncoimmunology 2018, 7(10):e1490854.

33. Hause RJ, Pritchard CC, Shendure J, Salipante SJ: Classification and characterization of microsatellite instability across 18 cancer types. Nat Med 2016, 22(11):1342-1350.

34. Le DT, Uram JN, Wang H, Bartlett BR, Kemberling H, Eyring AD, Skora AD, Luber BS, Azad NS, Laheru D et al: PD-1 Blockade in Tumors with Mismatch-Repair Deficiency. N Engl J Med 2015, 372(26):2509-2520.

35. Timmermann B, Kerick M, Roehr C, Fischer A, Isau M, Boerno ST, Wunderlich A, Barmeyer C, Seemann $\mathrm{P}$, Koenig $\mathrm{J}$ et al: Somatic mutation profiles of MSI and MSS colorectal cancer identified by whole exome next generation sequencing and bioinformatics analysis. PLoS One 2010, 5(12):e15661.

36. Hinshaw DC, Shevde LA: The Tumor Microenvironment Innately Modulates Cancer Progression. Cancer Res 2019, 79(18):4557-4566.

37. Li F, Sun Y, Huang J, Xu W, Liu J, Yuan Z: CD4/CD8 + T cells, DC subsets, Foxp3, and IDO expression are predictive indictors of gastric cancer prognosis. Cancer Med 2019, 8(17):7330-7344.

38. Cabrita R, Lauss M, Sanna A, Donia M, Skaarup Larsen M, Mitra S, Johansson I, Phung B, Harbst K, Vallon-Christersson $\mathrm{J}$ et al: Tertiary lymphoid structures improve immunotherapy and survival in melanoma. Nature 2020, 577(7791):561-565.

39. Vihervuori H, Autere TA, Repo H, Kurki S, Kallio L, Lintunen MM, Talvinen K, Kronqvist P: Tumorinfiltrating lymphocytes and CD8(+) T cells predict survival of triple-negative breast cancer. J Cancer Res Clin Oncol 2019, 145(12):3105-3114.

40. van der Leun AM, Thommen DS, Schumacher TN: CD8(+) T cell states in human cancer: insights from single-cell analysis. Nat Rev Cancer 2020, 20(4):218-232.

41. Nielsen SR, Schmid MC: Macrophages as Key Drivers of Cancer Progression and Metastasis. Mediators Inflamm 2017, 2017:9624760.

42. Shan X, Zhang C, Wang Z, Wang K, Wang J, Qiu X, Jiang T, Yang P: Prognostic value of a nine-gene signature in glioma patients based on tumor-associated macrophages expression profiling. Clin Immunol 2020, 216:108430.

43. Kim J, Bae JS: Tumor-Associated Macrophages and Neutrophils in Tumor Microenvironment. Mediators Inflamm 2016, 2016:6058147.

44. Zhou H, He Y, Li L, Wu C, Hu G: Identification novel prognostic signatures for Head and Neck Squamous Cell Carcinoma based on ceRNA network construction and immune infiltration analysis. Int J Med Sci 2021, 18(5):1297-1311.

\section{Tables}

Table 1: Pan-cancer data acquired from TCGA 


\begin{tabular}{|c|c|c|c|}
\hline $\begin{array}{l}\text { Cancer } \\
\text { type }\end{array}$ & Full name & $\begin{array}{l}\text { Tumor } \\
\text { samples }\end{array}$ & $\begin{array}{l}\text { Normal } \\
\text { samples }\end{array}$ \\
\hline ACC & Adrenocortical carcinoma & 79 & 0 \\
\hline BLCA & Bladder urothelial carcinoma & 414 & 19 \\
\hline BRCA & Breast invasive carcinoma & 1109 & 120 \\
\hline CESC & $\begin{array}{l}\text { Cervical squamous cell carcinoma and endocervical } \\
\text { adenocarcinoma }\end{array}$ & 306 & 3 \\
\hline $\mathrm{CHOL}$ & Cholangiocarcinoma & 36 & 9 \\
\hline COAD & Colon adenocarcinoma & 480 & 41 \\
\hline ESCA & Esophageal carcinoma & 162 & 11 \\
\hline GBM & Glioblastoma multiforme & 169 & 5 \\
\hline HNSC & Head and neck squamous cell carcinoma & 502 & 44 \\
\hline $\mathrm{KICH}$ & Kidney chromophobe & 65 & 24 \\
\hline KIRC & Kidney renal clear cell carcinoma & 539 & 72 \\
\hline KIRP & Kidney renal papillary cell carcinoma & 289 & 32 \\
\hline LGG & Brain lower grade glioma & 529 & 0 \\
\hline LIHC & Liver hepatocellular carcinoma & 374 & 50 \\
\hline LUAD & Lung adenocarcinoma & 535 & 59 \\
\hline LUSC & Lung squamous cell carcinoma & 502 & 49 \\
\hline MESO & Mesothelioma & 86 & 0 \\
\hline OV & Ovarian serous cystadenocarcinoma & 379 & 0 \\
\hline PAAD & Pancreatic adenocarcinoma & 178 & 4 \\
\hline PCPG & Pheochromocytoma and paraganglioma & 183 & 3 \\
\hline PRAD & Prostate adenocarcinoma & 499 & 52 \\
\hline READ & Rectum adenocarcinoma & 167 & 10 \\
\hline SARC & Sarcoma & 263 & 2 \\
\hline SKCM & Skin cutaneous melanoma & 471 & 1 \\
\hline STAD & Stomach adenocarcinoma & 375 & 32 \\
\hline TGCT & Testicular germ cell tumors & 156 & 0 \\
\hline THCA & Thyroid carcinoma & 510 & 58 \\
\hline
\end{tabular}




\begin{tabular}{llll} 
THYM & Thymoma & 119 & 2 \\
\hline UCEC & Uterine corpus endometrial carcinoma & 552 & 35 \\
\hline UCS & Uterine carcinosarcoma & 56 & 0 \\
UVM & Uveal melanoma & 80 & 0
\end{tabular}

\section{Figures}


A

Type 官 Normal 户 Tumor

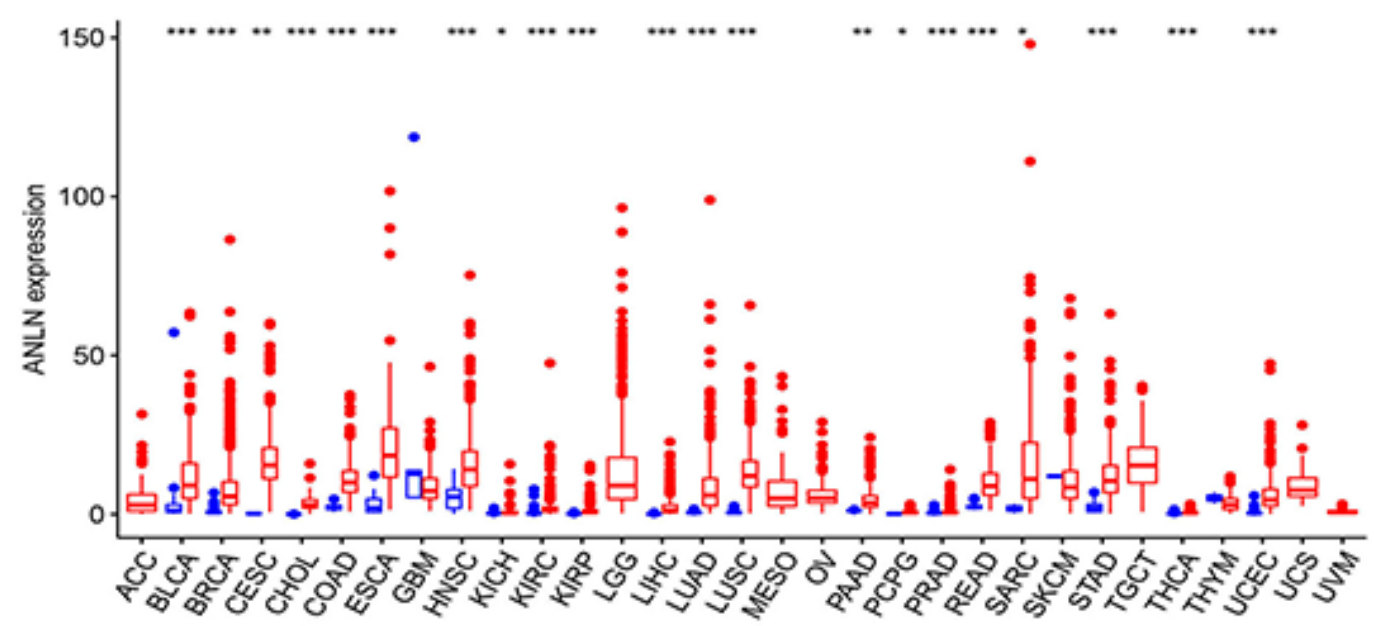

B

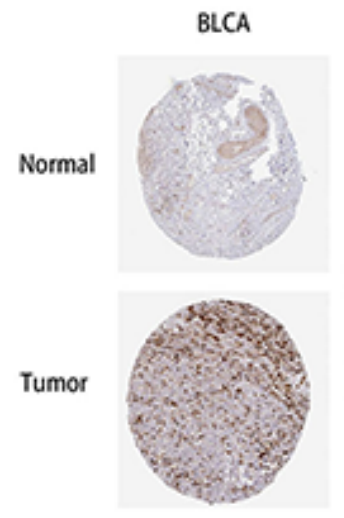

CAC

LUAD

ov

TGCT
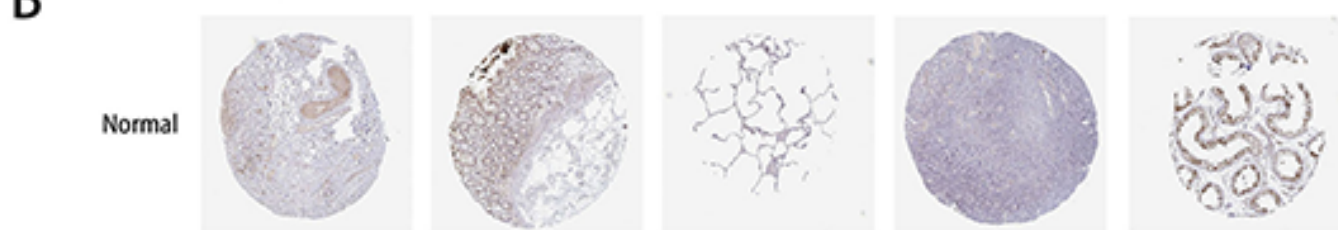

C
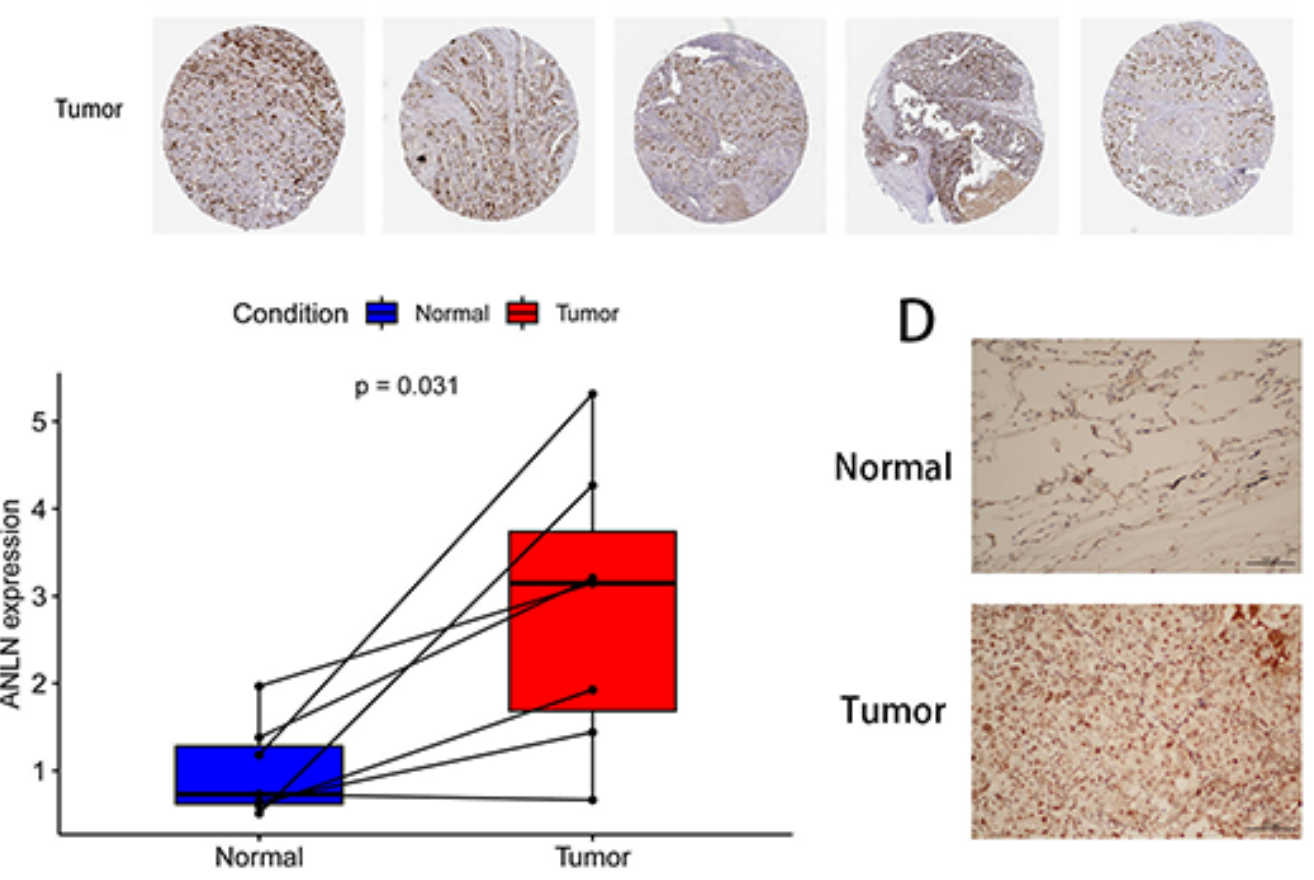

\section{Figure 1}

ANLN expression in pan-cancer. A: Boxplot of the gene expression of ANLN in cancers and normal tissues from TCGA database. B: Representative immunohistochemical staining of ANLN in colon, LUAD, PRAD normal and cancer samples, data from The Human Protein Atlas (THPA) database. ${ }^{\star} P<0.05 ;{ }^{\star \star P}<0.01$,

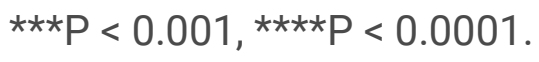



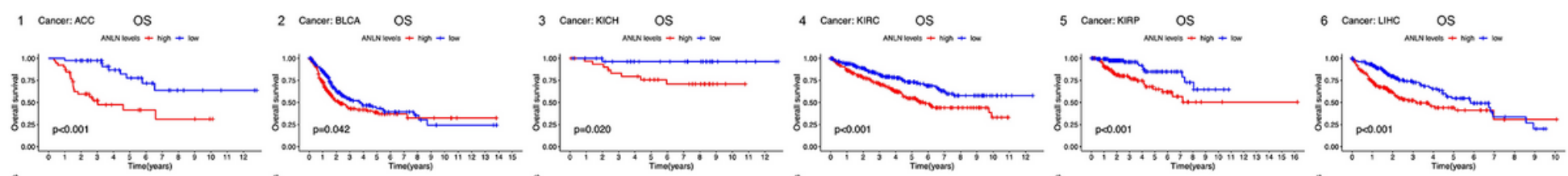

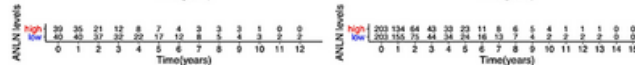

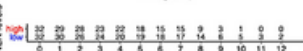

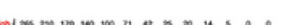

年

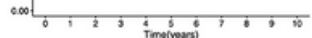
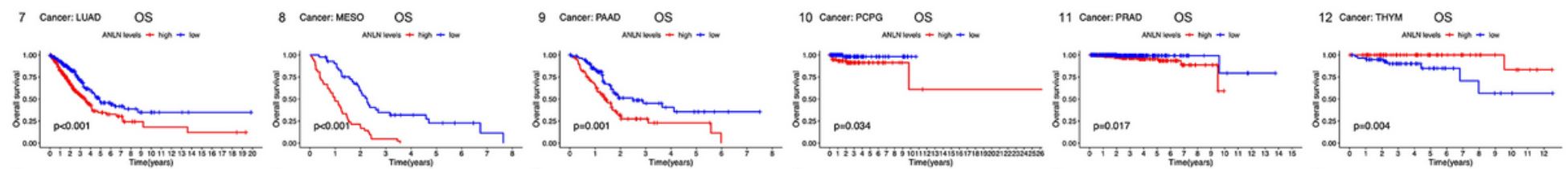

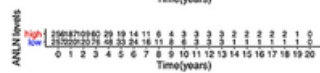

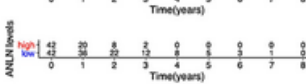

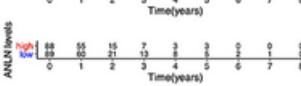
1)

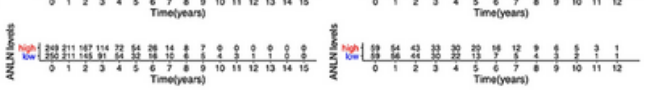
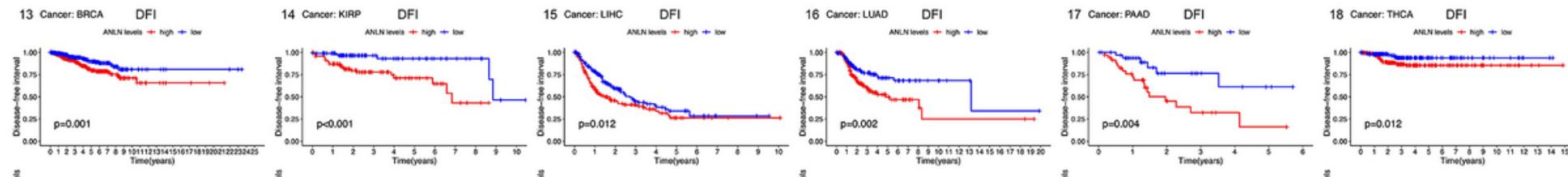
旁等

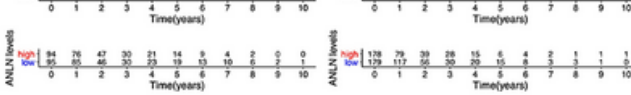

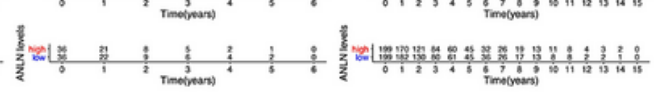
19 Cancer: $A C C \quad P F 1$ 20 Cancor: BLCA PFI
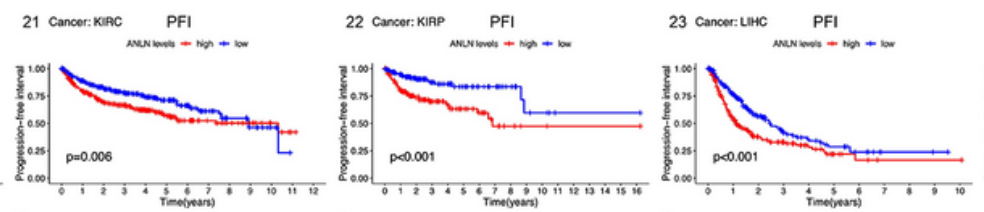
24 Cancoer: Lund PFI
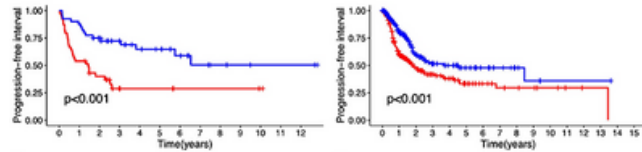

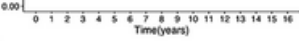

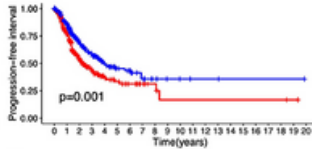

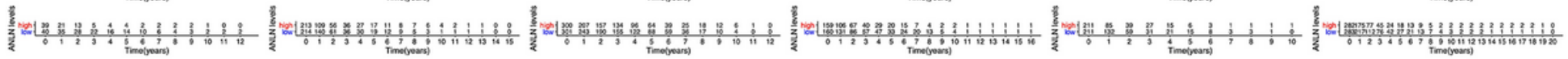

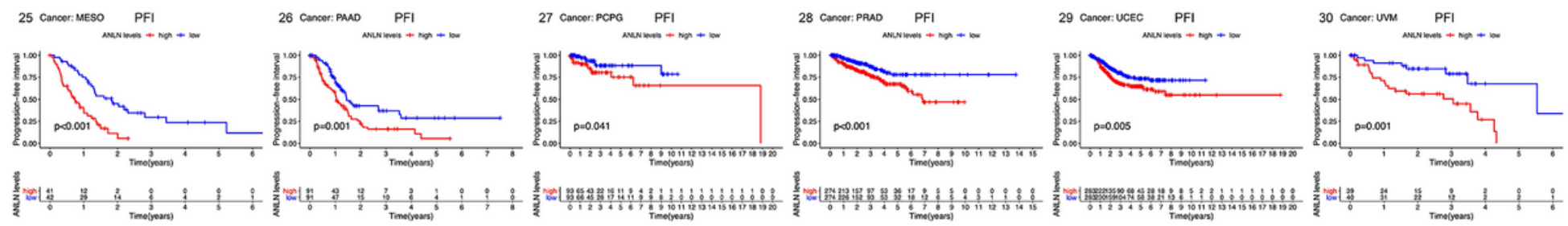

\section{Figure 2}

Kaplan-Meier survival curves comparing ANLN expression in pan-cancer. (1-12): Kaplan-Meier curves for patients' OS (overall survival) in 12 tumor types; (13-18): Kaplan-Meier curves for patients' DFI (diseasefree interval) in 6 tumor types; (19-30): Kaplan-Meier curves for patients' PFI (progression-free interval) in 12 tumor types. $\mathrm{P}<0.05$ was considered significant. 
A

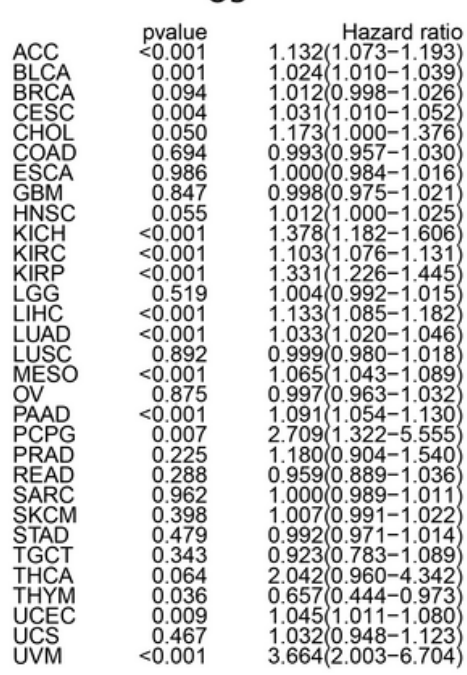

C

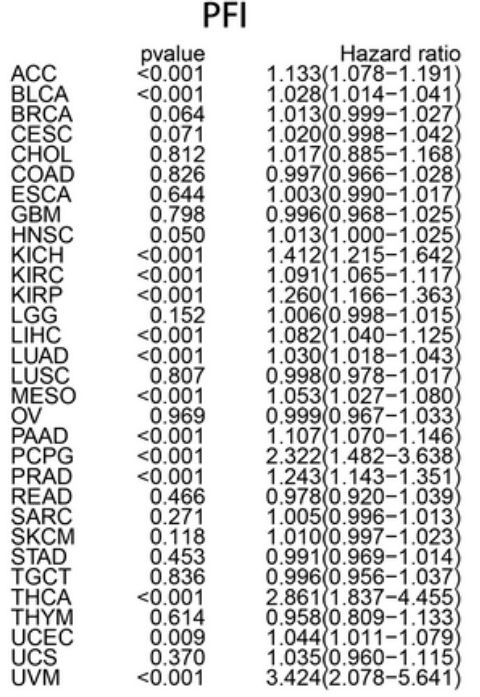

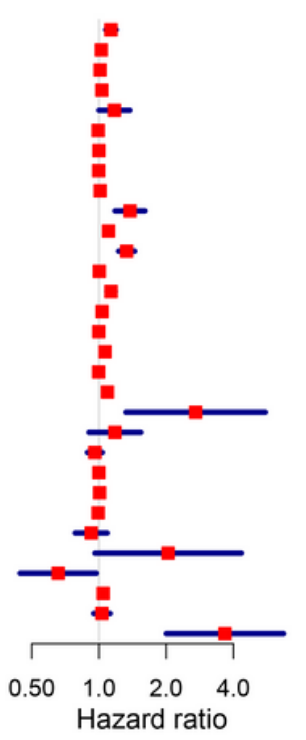

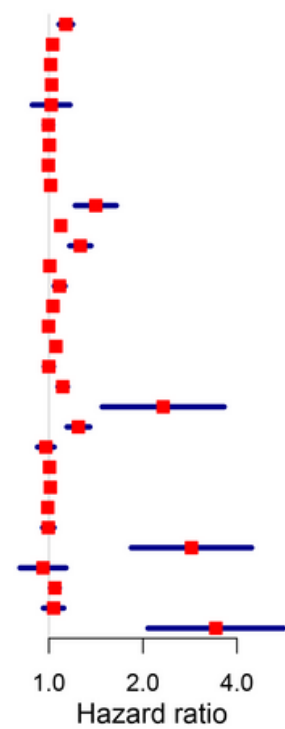

B

$\begin{array}{lc} & \text { pvalue } \\ \text { ACC } & 0.795 \\ \text { BLCA } & 0.631 \\ \text { BRCA } & 0.009 \\ \text { CESC } & 0.511 \\ \text { CHOL } & 0.241 \\ \text { COAD } & 0.896 \\ \text { ESCA } & 0.559 \\ \text { HNSC } & 0.765 \\ \text { KIIH } & 0.324 \\ \text { KIRC } & 0.701 \\ \text { KRP } & <0.001 \\ \text { LGG } & 0.057 \\ \text { LIHC } & 0.001 \\ \text { LUAD } & 0.035 \\ \text { LUSC } & 0.687 \\ \text { MESO } & 0.065 \\ \text { OV } & 0.481 \\ \text { PAAD } & 0.001 \\ \text { PCD } & 0.367 \\ \text { PRAD } & 0.002 \\ \text { PRAD } & 0.073 \\ \text { READ } & 0.973 \\ \text { SARC } & 0.113 \\ \text { SARD } & 0.075 \\ \text { TGCT } & 0.484 \\ \text { THCA } & <0.001 \\ \text { UCEC } & 0.437 \\ \text { UCS } & 0.389 \\ \text { UCS } & \end{array}$

DFI

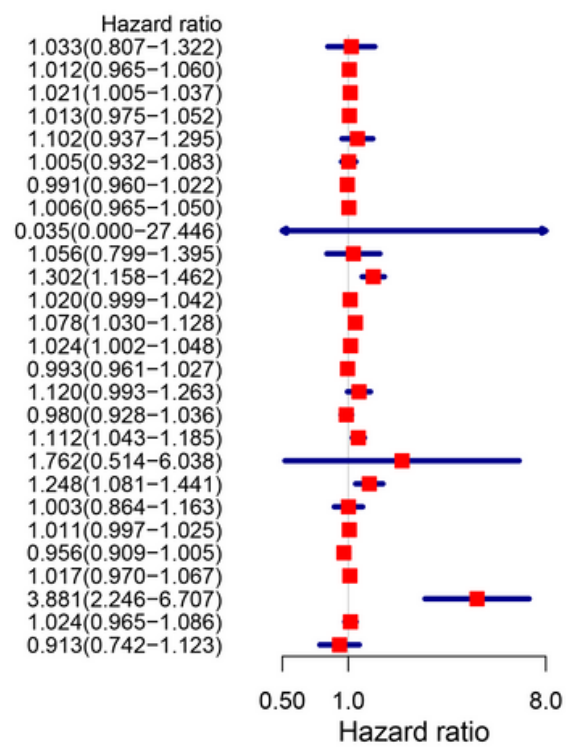

Figure 3

Forest plots of hazard ratios of ANLN in pan-cancer. A: Association between ANLN expression and OS; B: Association between ANLN expression and DFI; C: Association between ANLN expression and PFI. Cox regression analysis. $\mathrm{P}<0.05$ was considered significant. 
Cancer: ACC

Stage 自 Stagel 审 serage II 审 Stage III 审 Stage IV

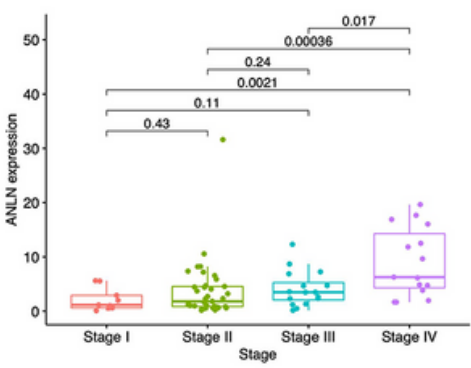

Cancer: KICH

Stage 审 Stage I 审 Stroge II 审 Stage III 审 Stage IV

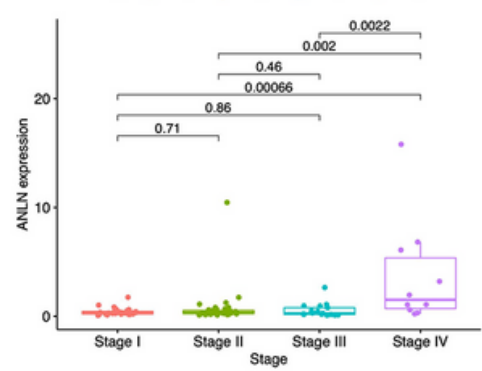

Cancer: LUAD

Stage 审 Stagol 审 Stage II 审 Stage III 由 Stago IV

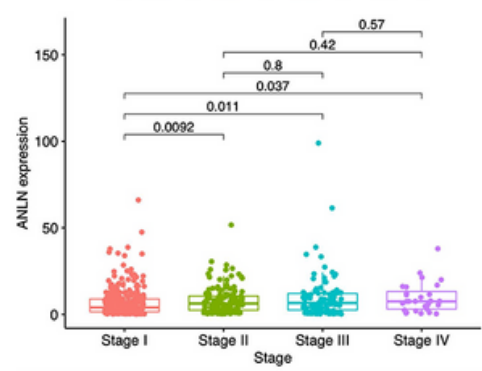

Cancer: BLCA

Stage 审 Suagel 自 Stage II 审 Stage III 审 Stage IN

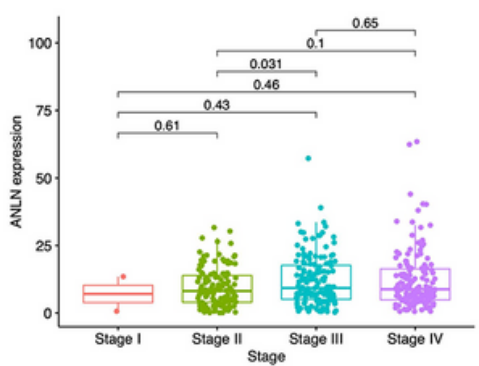

Cancer: KIRC

Stage 审 Stagel 由 Stage II 由 Stage III 审 Stage IV

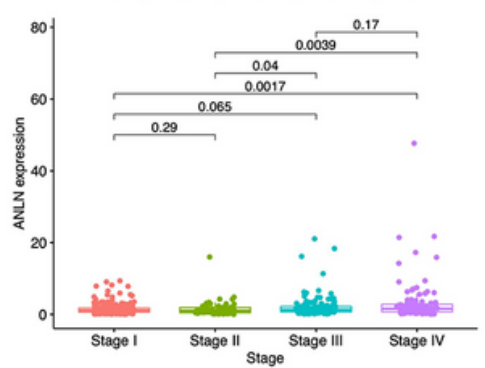

Cancer: THCA

Stage 审 Stasgo I 审 Stage II 审 Stage iII 审 Stage IV

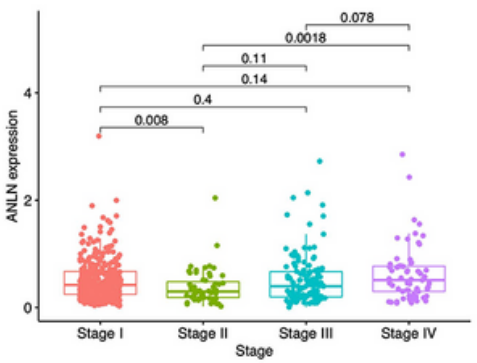

Cancer: BRCA

Stage 审 Staspe 1 审 Stage II 审 Stage III 审 Stage N

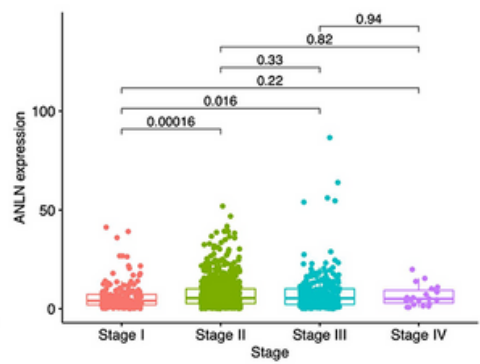

Cancer: KIRP

Stage 审 Stagel 审 Stage II 审 Stugo III 审 Stage IV

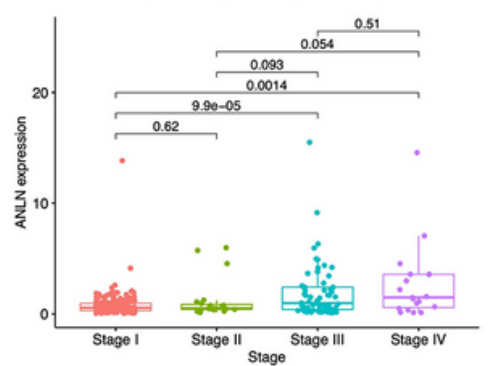

Cancer: ESCA

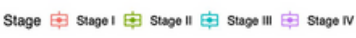

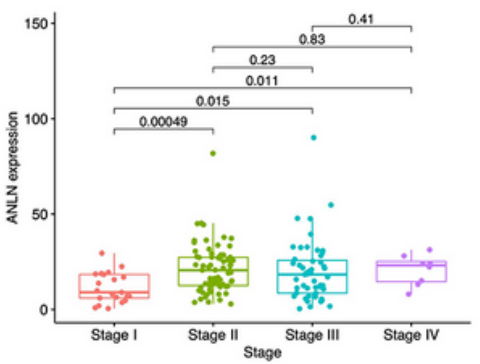

Cancer: LIHC

Stage 审 Stage I 审 Stage II 审 Stage III 审 Stugo IV

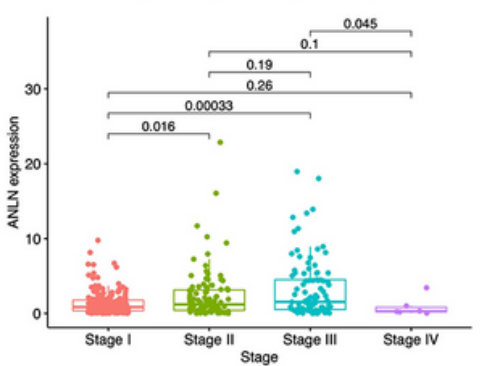

\section{Figure 4}

Association between ANLN expression and different TNM stages in patients with ACC, BLCA, BRCA, ESCA, KICH, KIRC, KIRP, LUAD, LIHC and THCA. P $<0.05$ was considered significant. 

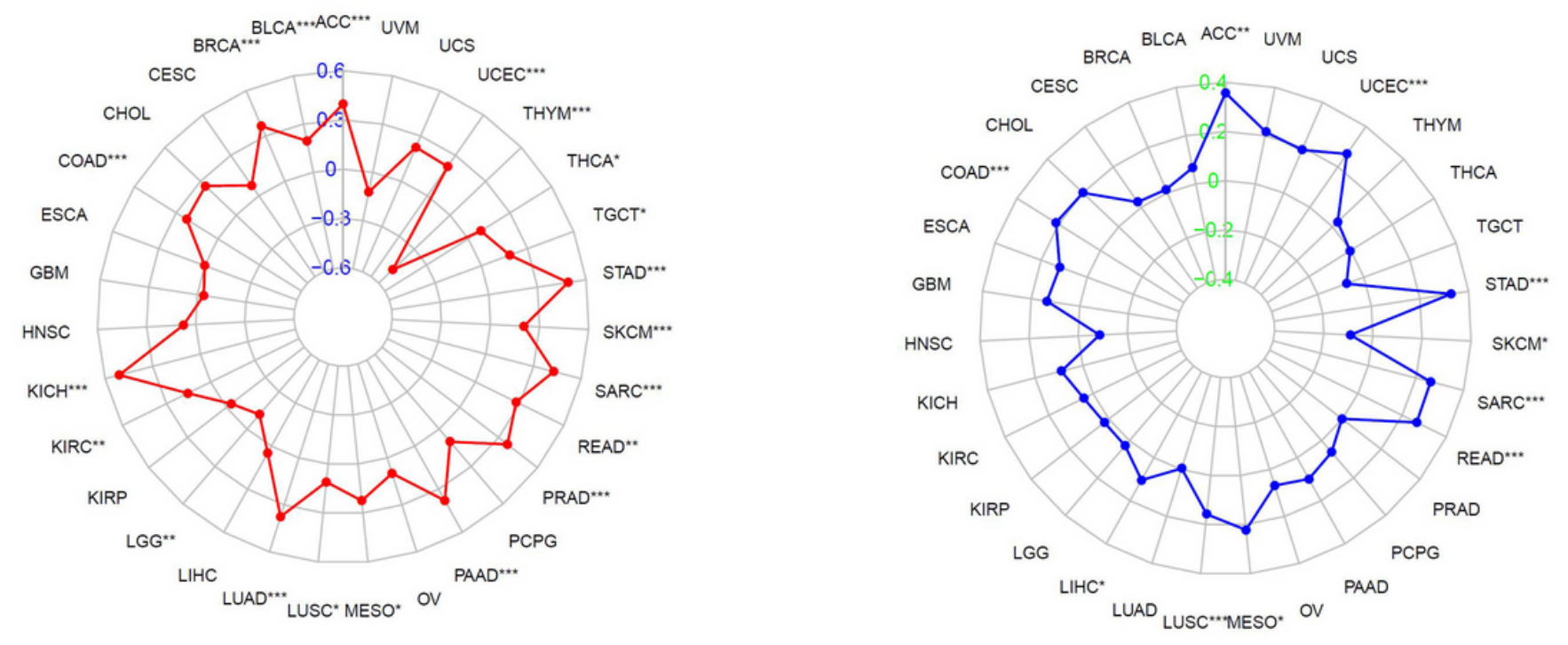

Figure 5

Associations between ANLN expression and tumor mutation burden (TMB) and microsatellite instability (MSI) across pan-cancer. A: Association between ANLN expression and TMB; B: Association between ANLN expression and MSI. ${ }^{*} \mathrm{P}<0.05, * * \mathrm{P}<0.01,{ }^{*} * \mathrm{P}<0.001$.
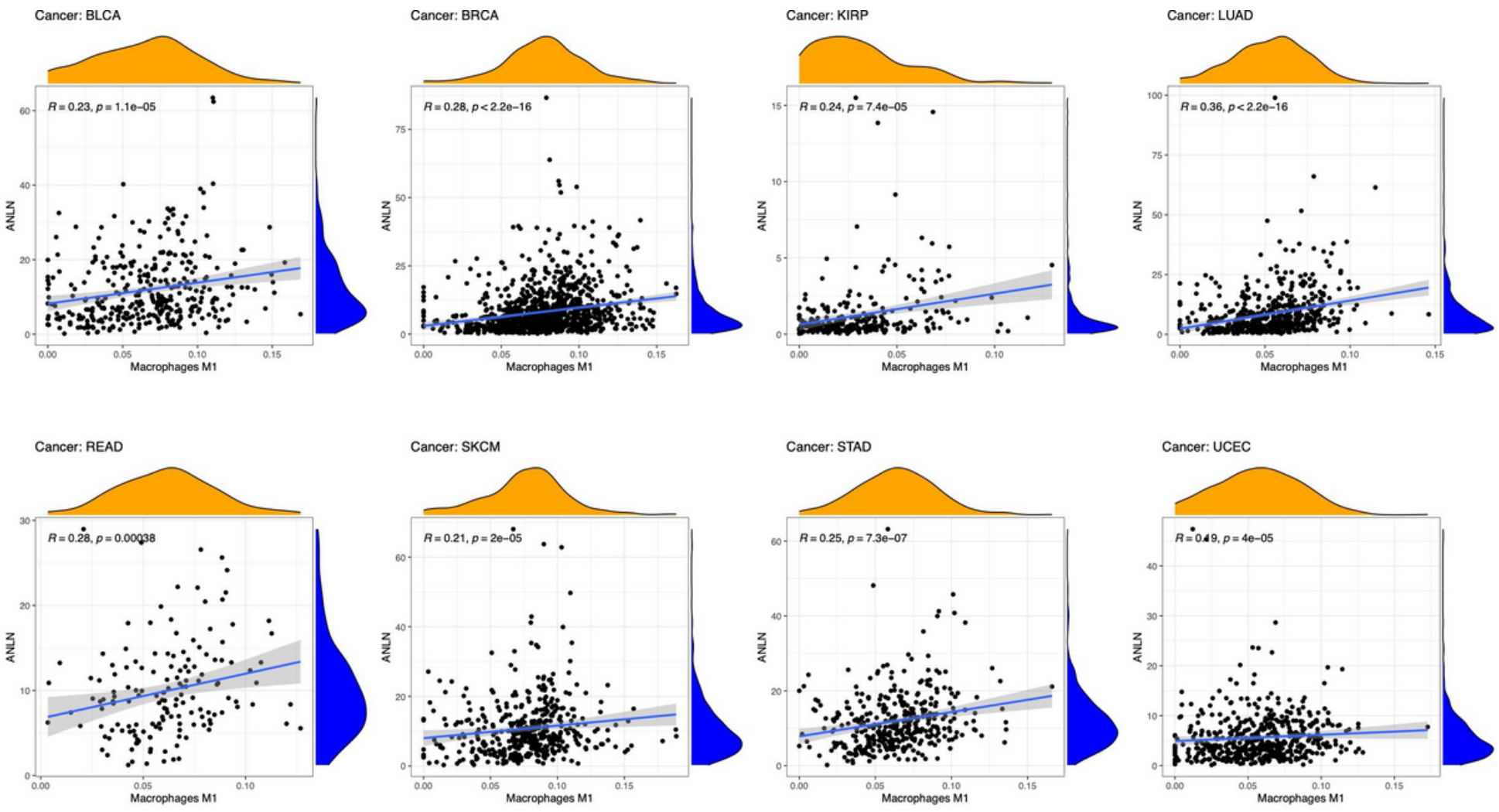

Figure 6 
Association between ANLN expression and Macrophages M1 in in patients with BLCA, BRCA, KIRP, LUAD, READ, SKCM, STAD and UCEC $(P<0.00001)$.
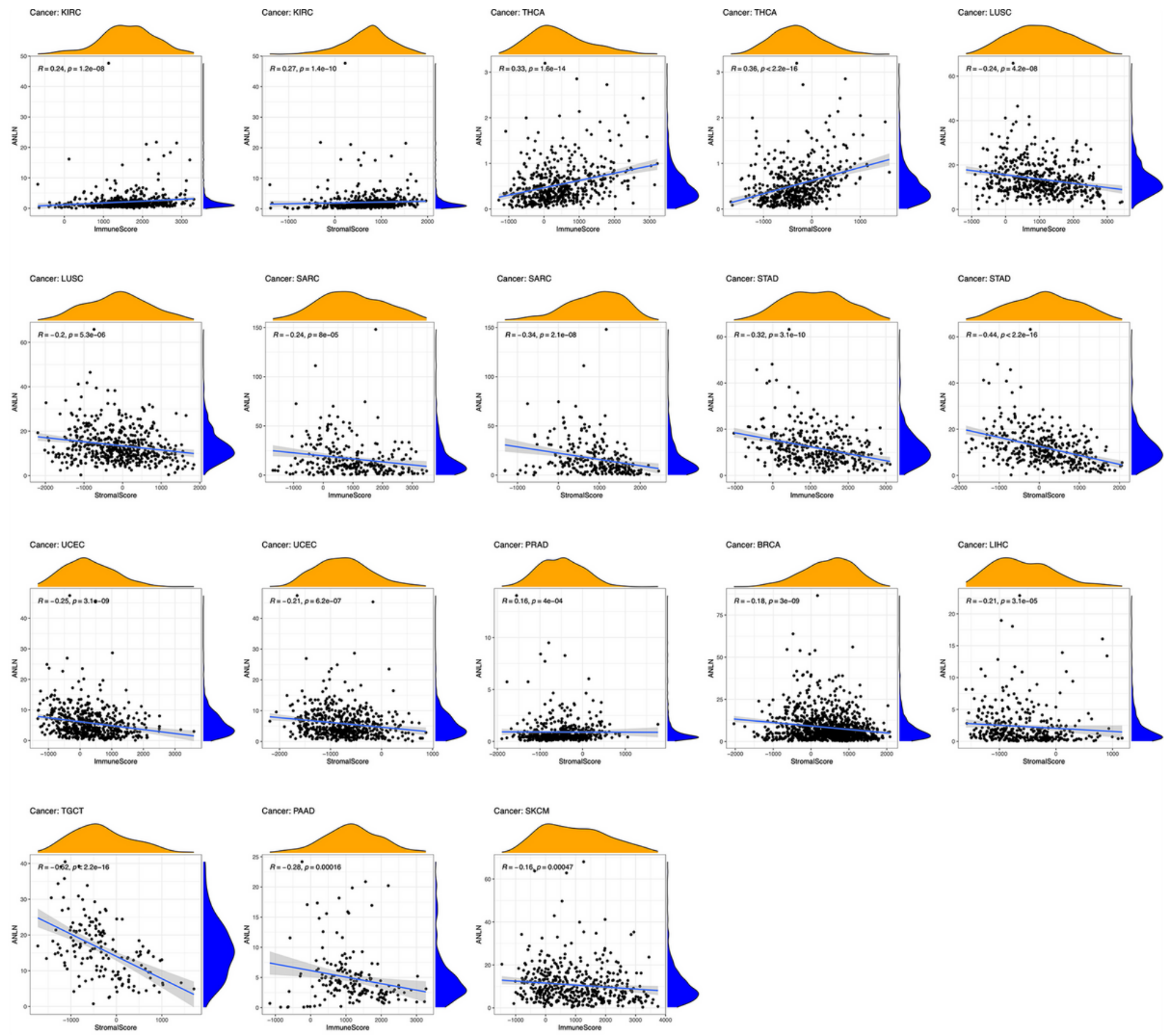

\section{Figure 7}

Association between ANLN expression and the immune and stromal cell ratio in patients' with KIRC, THCA, LUSC, SARC, STAD, UCEC, PRAD, BRCA, LIHC, TGCT, PAAD and SKCM. P $<0.05$ was considered significant. 


\section{Coexpression across cancer types}

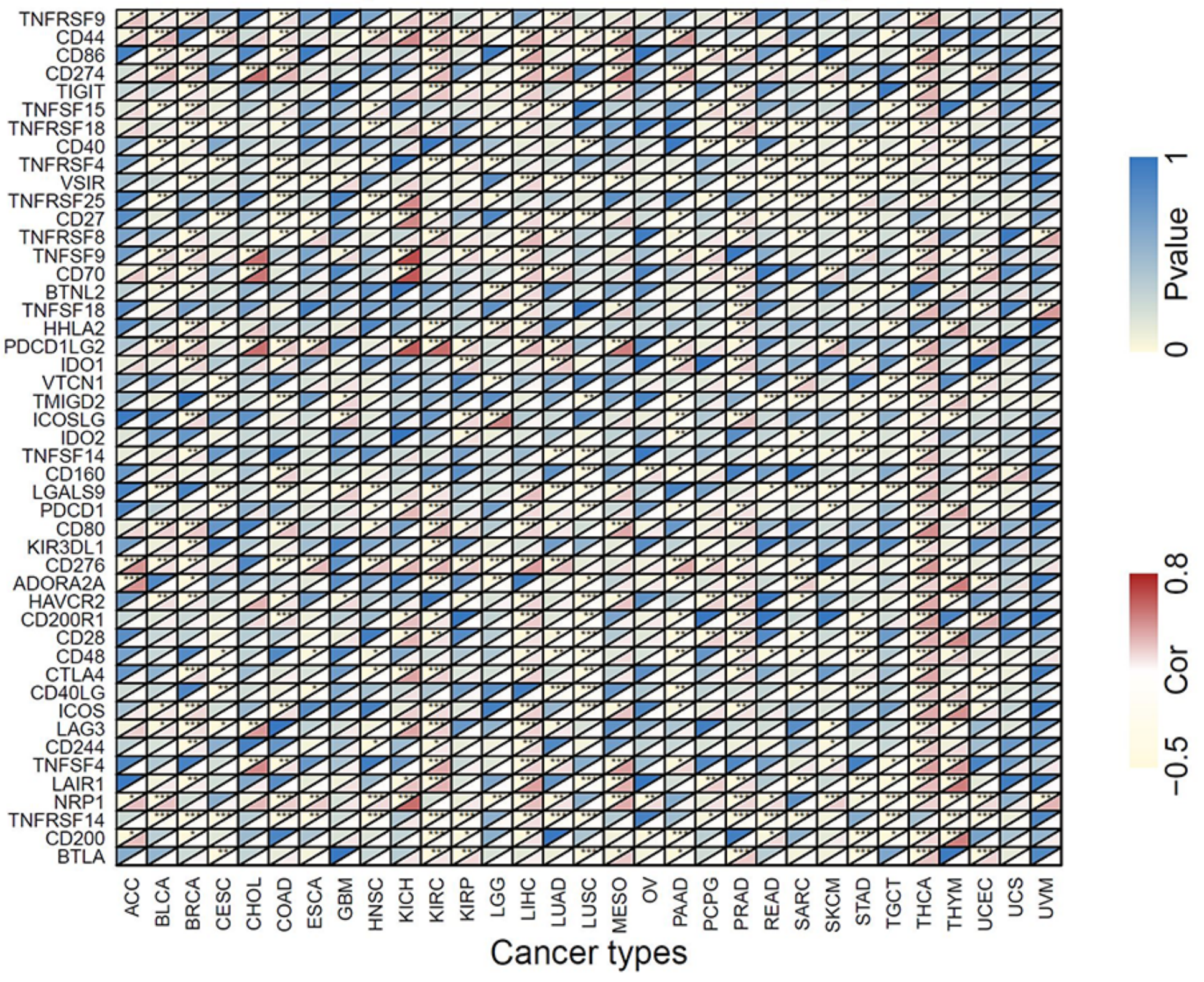

Figure 8

Association between ANLN expression and 47 common immune checkpoints gene levels in pan-cancer. *P $<0.05, * * \mathrm{P}<0.01, * \star * \mathrm{P}<0.001$. 
A
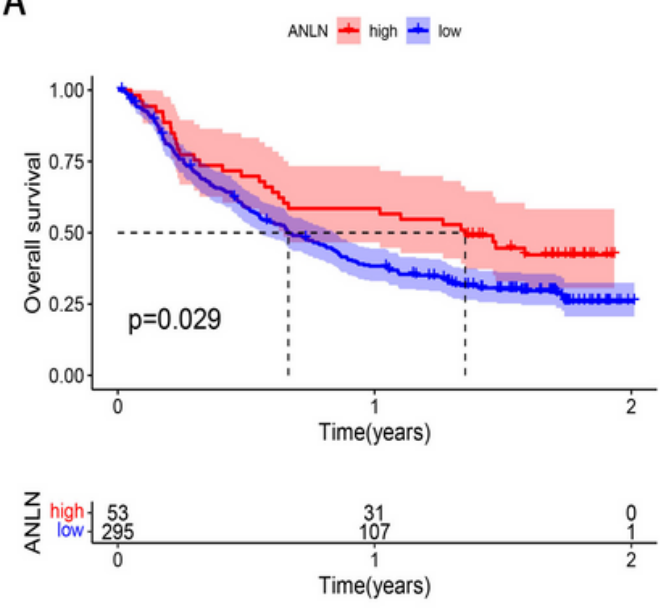

B

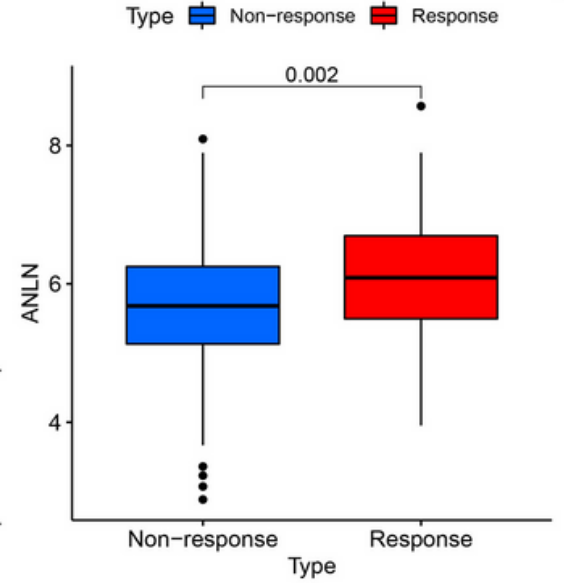

C

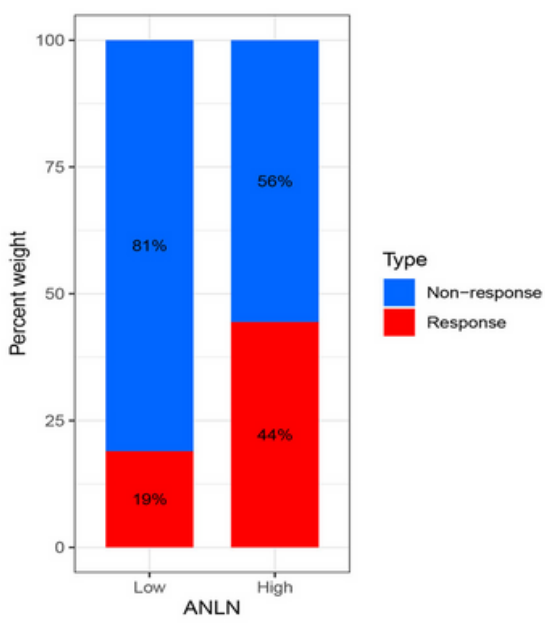

Figure 9

ANLN expression in the role of immunotherapeutic response prediction. A: Kaplan-Meier curves for patients' OS; B: Differences in ANLN expression between non-response and response types; C: The proportion of patients with response to PD-L1 blockade immunotherapy in low or high ANLN groups. $P$ $<0.05$ was considered significant.

\section{Supplementary Files}

This is a list of supplementary files associated with this preprint. Click to download.

- Supplementrayfigures.zip 